\title{
Regenerative Therapien punkten bei Knorpelschäden
}

Hatte der Knorpel Schaden genommen, galt lange Zeit die mechanische Knorpelglättung als Mittel der Wahl. Inzwischen ist die „Gelenktoilette“ in den meisten Fällen „out“. Für regenerative Verfahren spricht dagegen eine immer breitere Datenbasis.

\begin{abstract}
Die mechanische Knorpelglättung war in Deutschland einer der am häufigsten durchgeführten Eingriffe, wie Prof. Dr. Gunter Spahn, Klinik für Unfall-, Hand- und Wiederherstellungschirurgie, Universitätsklinikum Jena, im Rahmen der Sitzung „Knorpelregeneration und Knorpelreparatur" betonte. Laut Statistischem Bundesamt 2017 zählte das Knorpeldebridement mit mehr als 161.700 stationären Behandlungsfällen zu den 20 häufigsten Prozeduren in Deutschland.
\end{abstract}

》) Es gibt kaum Evidenz für die Wirksamkeit ungezielter Knorpelglättung bei Gonarthrose

Unter Berücksichtigung der ambulant durchgeführten sei die Zahl sicher doppelt so hoch - und das, nachdem der deutsche G-BA (Gemeinsamer Bundesausschuss) die ungezielte Knorpelglättung bei Gonarthrose 2015 für unwirksam erklärt hat. Schließlich gibt es kaum Evidenz für die Wirksamkeit. Bei Gonarthrose führt sie allenfalls kurzfristig, häufig aber zu gar keiner Besserung der Beschwerden; einen Stopp des Arthroseprozesses vermag sie nicht zu bewirken.

\) Bei Knorpeldefekten unter $1 \mathrm{~cm}^{2}$ Größe ist keine Therapie erforderlich

Quelle: Dr. Wiebke Kathmann, Springermedizin.de, basierend auf: 46. Kongress der Deutschen Gesellschaft für Rheumatologie (DGRh), 19.22. September 2018 Mannheim In einem eigenen Review von 2017 zu 40 relevanten Studien fanden Spahn et al. bei $20 \%$ der Patienten nach zwei Jahren eine Verbesserung der Kniescores um nicht mehr als 20 von 100 Punkten. Auch Rasterelekronenmikroskopische Untersuchungen spre- chen dagegen, dass durch die "Gelenktoilette" glatte Oberflächen geschaffen werden und somit der Reibungswiderstand verringert werden kann. Vielmehr besteht die Gefahr einer Schädigung des subchrondralen Knochens. Vor allem konnte in randomisierten Studien gezeigt werden, dass chondroplastische Verfahren deutlich besser abschneiden.

\section{Autologe Knorpelzelltransplanta- tion nicht nur bei Trauma}

Generell ist bei Knorpeldefekten unter $1 \mathrm{~cm}^{2}$ Größe keine Therapie erforderlich, bei größeren sollten bioregenerative Verfahren eingesetzt werden. Insbesondere die autologe Knorpelzelltransplantation (ACT) hat sich als effektiv erwiesen. Inzwischen wird sie nicht mehr nur bei traumatischen, sondern auch degenerativen Schäden des Gelenkknorpels eingesetzt, wie die Daten des Deutschen Knorpelregisters offenbaren: Die ACT wird nicht mehr nur bei isolierten Knorpelschäden mit stabilen Defekträndern durchgeführt, sondern auch bei fokalen degenerativen Defekten. Denn auch in dieser Situation vermag sie die Funktion zu verbessern sowie Schmerzen und Schwellung zu verringern; sie geht allerdings häufiger mit Komplikationen einher.

Laut PD Dr. Johannes Zellner, Klinik St. Josef, Universitätsklinikum Regensburg, ist eine gute Patientenselektion für den Erfolg der ACT entscheidend. Wichtige Parameter sind Größe des Defektes ( $>2,5 \mathrm{~cm}^{2}$ bei junge, aktiven Patienten, sonst $>3-4 \mathrm{~cm}^{2}$ ) und
Defektart (fokale bzw. isolierte Knorpelschäden). Begleitpathologien des Gelenkes, wie Achsenfehlstellungen, Kreuzbandinstabilitäten und Meniskuspathologien, sollten immer mitversorgt werden, so Zellner.

》) Bei größeren Knorpeldefekten hat sich die autologe Knorpelzelltransplantation als effektiv erwiesen

Das Verfahren selbst hat sich gegenüber früher stark vereinfacht. Heute wird üblicherweise eine Knorpelzellmatrix auf den Knorpeldefekt aufgebracht. Die Zellen weisen ein stabiles chondrogenes Potential auf. Nach der regenerativen Therapie kommt es über etwa 5-10 Jahre zu einem signifikanten und stabilen Anstieg der klinischen Funktionsscores. Negative Prädiktoren für das Outcome sind laut Zellner höheres Alter, weibliches Geschlecht, lange bestehende Beschwerden, mehrfache Voroperationen, Vorliegen mehrerer Defekte und eine patellofemorale Lokalisation. rheuma plus $2018 \cdot 17: 192$ https://doi.org/10.1007/s12688018-0225-8 (c) Springer-Verlag GmbH Austria, ein Teil von Springer Nature 2018 